\title{
Have Orbitals Really Been Observed?
}

\author{
Eric R. Scerri \\ Department of Chemistry and Biochemistry, University of California, Los Angeles, Los Angeles, CA 90095; \\ scerri@chem.ucla.edu
}

The purpose of this article is to comment on the pedagogical ramifications of the recently claimed observation of $d$ orbitals and the way in which these results were first announced and subsequently reported (1-6).

Atomic orbitals - their use in writing electronic configurations and as a means of rationalizing so much of modern chemistry-have, rightly or wrongly, become ubiquitous in textbooks as well as the research literature. Orbitals are part of the lingua franca of chemistry. They represent one of chemistry's major paradigms, to use a much abused term (7). Surely it is essential that claims to having arrived at a new understanding of such a crucially important educational concept should be subjected to close scrutiny. It is also essential for chemical educators at all levels to take note of these developments in order to adjust their teaching accordingly if such adjustments are necessary. At the very least, educators should take some time to reflect on the meaning of such an important concept as an atomic orbital when it is claimed that, contrary to previous beliefs, they have now been observed for the first time.

\section{The Report and Its Aftermath}

As often seems to be the case with startling new discoveries, it appears that the manner in which this finding was communicated has given rise to a certain amount of confusion, to say the least. What is surprising, however, is that in this particular case the initial mode of communication was not a press conference, as in the infamous case of cold fusion, but the publication of an article in the prestigious journal Nature.

I believe that the authors of this article come close to making the claim that $\mathrm{d}$ orbitals have been literally observed when they write (1),

The correspondence between our experimental map and the classical diagrams of $\mathrm{d}_{z}^{2}$ orbitals sketched in textbooks is striking. All our difference maps show strong nonspherical charge distributions around the copper atoms, with the characteristic shape of $\mathrm{d}$ orbitals.

What exacerbated the situation is an accompanying editorial article with fewer, if any, reservations concerning the identification of the images obtained with textbook orbitals. The headline caption for the editorial begins (2),

The classic textbook shape of electron orbitals has now been directly observed.

The main body of the editorial states that,

For the first time the striking shape of some of the electron orbitals is revealed experimentally. The paper by Zuo et al. is remarkable because the quality of their charge-density maps allows, for the first time, a direct experimental 'picture' to be taken of the complex shape of the $\mathrm{d}_{z}{ }^{2}$ orbital.

In addition, the front page of this issue of Nature featured the simple words "Orbitals observed" in large bold letters, alongside some images taken from the primary article. Not surprisingly, some more popular accounts have not expressed any caution whatsoever in reporting the new findings. For example, a Web page produced by a leading popular science magazine boldly claims (3),

The idea of orbitals has long proved useful for describing atoms and their interactions mathematically, but not physically. Now all that's changed. Researchers at Arizona State University recently published in Nature the first true images of atomic orbitals in $\mathrm{Cu}_{2} \mathrm{O}$, a crystal called cuprite.

An article appearing in Chemical and Engineering News begins (5)

Remember the really neat-looking d-orbital from freshman chemistry? The one that looks like a three dimensional figure 8 with a doughnut around its midsection? Well, it's just been experimentally observed by Scientists at Arizona State University at Tempe.

It should be borne in mind that this journal is directed at members of the American Chemical Society and not just members of the interested public. ${ }^{1}$

\section{Theoretical Issues}

Let me now turn to the theoretical status and limitations of orbitals and why orbitals cannot possibly be observed. Atomic orbitals are mathematical constructs and strictly speaking are only genuine wave functions in one-electron systems such as the hydrogen atom. In many-electron atoms orbitals serve as a useful approximation as employed, for example, in the aufbau process to explain the features of the periodic system in an approximate manner. Atomic orbitals also serve as a means of classifying spectroscopic transitions in the study of atomic spectra (8).

The orbital approximation is the basis of a great deal of the work conducted in quantum chemistry, but here it is recognized that orbitals are mathematical constructs and do not possess any independent physical status. According to quantum mechanics the assignment of four quantum numbers to each electron in a many-electron atom, which is another way of characterizing an orbital, is an approximation. In fact, only the atom as a whole possesses well-defined stationary states and these states are characterized by the vectorial coupling of individual electronic momenta, with different coupling schemes operating depending on whether spin-orbit coupling is significant.

Moreover, the usual textbook statements that refer to particular numbers of electrons in particular orbitals, such as $1 \mathrm{~s}, 2 \mathrm{p}$, or $3 \mathrm{~d}$ orbitals, are in strict violation of the idea of indistinguishability of electrons. The requirement that electrons be indistinguishable from one another comes from the Pauli principle, which maintains that a physically admissible wave function for a system of fermions must be antisymmetric with 
respect to exchange of the coordinates of any pair of those fermions. The indistinguishability of electrons is an immediate corollary of this basic fact. These shortcomings of the orbital approximation are counteracted, to some extent, by means of permutation of all the electrons in the course of HartreeFock calculations. If one insists on retaining a physical picture, this would correspond to regarding each electron as being in every single orbital simultaneously. But even this permutation procedure does not succeed in including so-called dynamic correlation between electrons and so doing requires the use of more sophisticated mathematical techniques that go beyond the orbital approximation.

Of course the orbital model remains enormously useful as a first approximation and lies at the heart of much of computational chemistry; but it is just that, a model, as computational chemists and physicists are well aware. According to accepted current theory atomic orbitals serve merely as basis setsthat is, as types of coordinate systems that can be used to expand mathematically the wave function of any particular physical system. Just as the coordinate system of $x, y$, and $z$ used to describe any particular experiment in classical physics is unobservable, so too atomic orbitals are completely unobservable even in principle.

\section{Observational Aspects}

What can be observed, and frequently is observed in experiments, is electron density. In fact, the observation of electron density is a major field of research in which several monographs and review articles have been written (9). However, an examination of this literature shows that researchers consistently discuss the observation of electron density but not of orbitals.

In the recently reported experiments, Zuo et al. fitted experimental X-ray and electron diffraction data to a model called multipole refinement. This method does not assume an actual sum of atomic contributions but fits the data by an expansion in terms of radial functions multiplied by spherical harmonics on various centers. The result is a charge density, which is then compared to that obtained as a superposition of spherical atomic contributions assuming that the compound is perfectly ionic. The density-difference map is thus obtained as the difference between the experimental fit and the spherical or purely ionic fit.

In general, the result of experiments of this type and their subsequent analysis is the total electron density, which can be and often is analyzed directly. Moreover, in the case of molecular crystals or metals there is of course no assumption that the compound is ionic.

I do not deny that the techniques reported may have provided an image of overall electron density in the copper compounds in question. My aim is only to question whether "The classic textbook shape of electron orbitals has now been directly observed" (2), to quote again the editorial in Nature, and the suggested linking of the images obtained with textbook $3 \mathrm{~d}$ orbitals in the primary paper.

Although I may have appeared to absolve the primary authors themselves from making the identification between the images they obtained and textbook orbitals, I believe the situation to be more complicated. If these authors had wished to be cautious in the presentation of their findings, it seems a little odd that they should have expressed their findings in such a suggestive and, as it has turned out, rather misleading manner. It is also surprising that in all their quoted remarks, which have appeared in other magazines and even daily newspapers, the authors have done nothing to deny or at least diminish the reports that they have in fact directly observed some textbook orbitals. If this is due to a conflation of the terms "electron density" and "orbital" I suggest that it should be resisted, since such terms have precise meanings, which should be kept distinct.

\section{Advice to Educators}

My advice to chemistry educators is to avoid being seduced by the recent reports and not to revise their long-held view that atomic orbitals are just mathematical constructs. Textbook orbitals will never be observed, although they will probably retain their great usefulness in many branches of science. Atomic orbitals should not be identified with electron density, because doing so can only lead to further confusion in such areas as computational chemistry.

In this rapidly expanding area major advances have taken place in recent years. Whereas the self-consistent field method for performing atomic and molecular calculations was originally implemented through various orbital methods, the density functional approach, which considers electron density instead of orbitals and wave functions, has become increasingly important-and in fact computationally superior, in the case of large systems. Many excellent reviews have been published on the relationship between the traditional orbital ab initio methods and the more recent density functional approaches (10). Some of these approximation methods, such as the Kohn-Sham theories, for example, involve mixtures of the two approaches, whereas the Sham theory uses only density functionals. The point I am trying to emphasize is that it is important to distinguish between orbitals and electron density at advanced as well as elementary levels of chemical education. The recent claim to having observed orbitals conflates the terms density and orbitals and only serves to blur this important distinction.

\section{Direct and Indirect Observation}

Readers might be wondering how this situation relates to the fairly ubiquitous claims regarding the observation of atoms using scanning tunneling microscopy (STM) and atomic force microscopy (AFM). Of course atoms are not being directly observed in these studies, since all that is measured is the flow of current across a tip, or the force that the tip exerts when passing across a surface. Is the question of the putative observation of orbitals analogous?

My response to this question will be in two parts. The situation with the recent reports is analogous in the sense that electron density is also being indirectly observed (in fact, more indirectly than atoms, since the technique involves subtracting a reference-state density, which is not the case in STM and AFM studies). ${ }^{2}$ The second part of my response is that in the case of orbital observation there is simply no analogy with the case of atoms. 
It is not just that orbitals cannot be directly observed. They cannot be observed, period. Whereas there is nothing in the formalism of quantum mechanics to forbid the observation of atoms (or electron density), the same theory dictates that orbitals are not observable. Of course the theory may be incorrect; but if so, one would need to obtain some independent evidence for its breakdown. The authors of the Nature article clearly do not believe that they have refuted quantum mechanics.

\section{Conclusions}

As is often the case in scientific research, the appeal of realism seems to be irresistible in some quarters and this is perhaps why the reports were not seriously challenged —or at least, no objections were raised in letters to the editor of any of the magazines that carried the story. ${ }^{3}$ But it is surely essential to be more discerning in attributing physical reality to entities that are defined theoretically and that the theory itself informs us do not exist physically. It is also rather unfortunate that popular science journals from which many chemical educators rightly draw inspiration as well as information should have misrepresented the recent findings. But as I suggested earlier this is not entirely surprising, in view of the way the results were first communicated.

In case anyone is under the impression that the claimed observation of orbitals is just a passing fad, I should draw attention to another recent article entitled "Seeing Molecular Orbitals" (11). Here similar kinds of conceptual errors are made in conflating the terms orbital and electron density, although there is at least no claim to having observed textbook orbitals.

Finally, I might add that since beginning to write this article I have become aware of two groups of researchers who have made related objections to the original article in Nature as well as the accompanying editorial. Spackman and other senior colleagues in the International Union of Crystallography have pointed out what they see as glaring errors in the Nature editorial by Humphreys (12). More recently, a group in Germany led by Schwarz have raised numerous objections against the primary article, including some comments similar to mine involving the nature of atomic orbitals (13). However, to the best of my knowledge, nobody has yet published a categorical objection to the very notion of observing orbitals as I am doing here, although I began doing so in an editorial in Foundations of Chemistry (14).

\section{Acknowledgments}

I acknowledge the help of reviewers in formulating a description of the recent experiments performed on copper compounds and other helpful suggestions. I also thank Philip
Coppens (Buffalo), Barry de Boer (Pennsylvania), John Spence (Arizona State), and Colin Humphreys (Cambridge) for discussion on experimental aspects of observing electron density, and several quantum chemists for discussion on the theoretical status of atomic orbitals. They are Peter Gill (Nottingham), Brian Sutcliffe (Brussels), Peter Taylor (San Diego), David Cook (Sheffield), and Eugen Schwarz (Siegen).

\section{Notes}

1. In fact, the same magazine rated the "observation" of orbitals as one of the five most significant events in chemistry for the year 1999 (6). The newsletter for the American Physical Society (APS News 2000, 9 [4]) likewise described the event as one of the highlights of the year in physics for 1999.

2. The subtraction of reference-state density is not an essential feature of the experimental electron density technique but a particular way of analysis, which was used, for example, in the study by Zuo et al. (1).

3. This was not for want of trying. My letters and those of others to these editors were all denied publication.

\section{Literature Cited}

1. Zuo, J. M.; Kim, M.; O’Keeffe, M.; Spence, J. C. H. Nature 1999, 401, 49-52.

2. Humphreys, C. J. Nature 1999, 401, 21-22.

3. Leutwyler, K. Observing Orbitals; http://www.sciam.com explorations/1999/092099cuprite (accessed Sep 2000).

4. Yam, P. Sci. Am. 1999, 281 (Nov), 30.

5. Jacoby, M. Chem. Eng. News 1999, 77 (36), 8.

6. Zurer, P. Chem. Eng. News 1999, 77 (48), 38.

7. Scerri, E. R. J. Chem. Educ. 2000, 77, 522-525.

8. Condon, E. U.; Shortley, G. H. The Theory of Atomic Spectra; Cambridge University Press: Cambridge, 1935.

9. Electron Distribution and the Chemical Bond; Coppens, P.; Hall, M. B., Eds.; Plenum: New York, 1982. Coppens, P. X-ray Charge Densities and Chemical Bonding; Oxford University Press: Oxford, 1997. Coppens, P. Annu. Rev. Phys. Chem. 1992, 43, 663-692.

10. Gill, P. M. W. In Encyclopedia of Computational Chemistry, Vol. 1; von Ragué Schleyer, P., Ed.; Wiley: Chichester, 1998; pp 678-689.

11. Pascual, J. I.; Gómez-Herrero, J.; Rogero, C.; Baró, A. M.; Sánchez-Portal, D.; Artacho, E.; Ordejón, P.; Soler, J. M. Chem. Phys. Lett. 2000, 321, 78-82.

12. Spackman, M. A.; Howard, J. A. K.; Destro, R. Int. Union Crystallogr. Newslett. 2000, 8, 2.

13. Wang, S. G.; Schwarz, W. H. E. Angew. Chem., Int. Ed. Engl. 2000, 39, 1757-1762.

14. Scerri, E. R. Foundations Chem. 2000, 2, 1-4. 